\title{
Pre-Analysis Plan for Daily Income, Labor Supply and Multiple Accounts: A Mobile Money Field Experiment with Micro-Entrepreneurs in Malawi*
}

\author{
Shilpa Aggarwal ${ }^{\dagger}$ \\ Valentina Brailovskaya \\ Jonathan Robinson ${ }^{\S}$
}

March 1, 2018

This document pre-specifies key aspects of the analysis for a randomized evaluation on the impact of the provision of simple savings technologies on the savings and labor supply decisions, and risk-coping abilities of low-income, self-employed individuals. Our study is set in the city of Blantyre, Malawi. Note that data collection for this project began in February 2017; however, we have not yet begun analysis of any post-treatment data at the time of this writing.

\section{Experimental Design}

Our sample consists of approximately 800 entrepreneurs in Blantyre, Malawi. This sample was randomly divided into a control group, and two groups which received savings devices. The first of these received simple metal boxes with a lock (the "lockbox" group), and the other received mobile money (henceforth, MM) accounts. Within the lockbox and MM groups, we randomly sampled individuals to be offered either a single account, or multiple accounts.1 1 The MM accounts are "as-is", with the additional feature of all withdrawal fees

${ }^{*}$ This research protocol was approved by the IRBs of UCSC, ISB, and the Malawi National Commission for Science and Technology.

†Indian School of Business, email: shilpa_aggarwal@isb.edu

$\ddagger$ University of California, Santa Cruz, email: vbrailov@ucsc.edu

§University of California, Santa Cruz, email: jmrtwo@ucsc.edu

${ }^{1}$ Individuals in the multiple lockbox group may take up to 3 boxes, while individuals in the multiple mobile money group may receive up to 2 accounts. The reason we offer only 2 accounts is because only 2 sim cards can be used simultaneously in the phones we use in the study. 
reimbursed to the participants for the duration of the study.

In addition, all study arms, including the control group, have been cross-randomized into a High-Frequency (HF) phone survey treatment. For two distinct 6-8 week-long periods over the course of the study, respondents in this treatment will be called once or twice weekly and surveyed regarding a number of outcomes, including labor supply, business investment, savings, cash flow, and household shocks.

In order to facilitate the phone survey as well as the MM treatment, respondents in these treatment arms had to be provided with cell phones. To eliminate the confounding effect of receiving a cell phone, all 800 individuals in the study were given cell-phones.

\section{Sampling}

Our experimental sample is drawn from a census of small businesses in 75 randomly selected enumeration areas in the city of Blantyre, Malawi. All businesses were included in the census. In total, 9,848 businesses were identified, of which $82 \%$ were classified as small businesses (not supermarkets or wholesale stores). A random sample of 3,457 small businesses were surveyed in order to collect basic information about their business. Using the information collected in this survey, about a third of the sample was deemed ineligible to participate in our study based on a set of 6 exclusion criteria. From our final list of 1,937 eligible participants who consented to participate in the study, 801 were randomly selected to participate. The study oversamples businesses which are connected to the electricity grid, as this sample is also being used for a companion paper on the impact of electricity outages on business decisions. Specifically, $35 \%$ of the sample is connected to the grid, compared to $26 \%$ in the universe of businesses. All regressions will be weighted to account for this.

\section{Outcomes and hypotheses}

\subsection{Main Outcomes}

We will utilize two main surveys to measure effects. First, half of the sample will take part in high frequency phone surveys which will allow us to examine outcomes at the daily or weekly level. Second, the entire sample will participate in monitoring surveys, which will measure outcomes over a longer look-back period. The surveys will include many outcomes. We include here the key outcomes we expect to explore.

- Savings: We will measure savings in the experimental savings product (lockbox or mobile money account), as well as total savings across all sources (including, for example, 
money saved in savings groups or at home). We plan to examine effects on withdrawals and deposits separately, and on the frequency and value of usage. We will also examine the account balance itself, though this will be a secondary outcome since the balance will understate usage if there are regular deposits and withdrawals.

- Credit: We will measure effects on borrowing behavior from formal and informal lending sources.

- Labor supply: We will measure daily labor supply, in the primary business as well as in any other occupations/farming activities

- Household expenditures: We will measure household expenditures on a list of aggregated expenditure categories. Potentially, we expect to see a reduction in expenses on temptation goods, and an increase in expenses on items such as education and preventive health, i.e., goods that provide no immediate gratification but may require saving up.

- Business investment, revenues, and profits: Our sample is made up entirely of microentrepreneurs, likely with little separation between business and personal finances. We will analyze several measures of business success, including inventories, investment, revenues, and profits.

\subsection{Surveys}

All outcomes will be measured using both HF and monitoring surveys. It is possible that the administration of the HF Surveys could be a treatment in and of itself since the respondents are asked to reflect on their financial decisions at least once a week. To measure the effects of frequent surveying, we will compare outcomes between the phone survey and non-phone survey groups at monitoring checks.. Finally, the last monitoring survey (i.e. endline) will be used to gather qualitative information on pathways through which the effects were realized as well as challenges faced with the products offered.

\section{Heterogeneity}

We plan to conduct heterogeneity on several characteristics, that we think may affect treatment effects. In particular, we expect:

- Effects of mobile money will be bigger for people who are closer to a mobile money agent. 
- Effects of both mobile money and lockboxes will be smaller for people with pre-existing access to financial instruments.

In addition, based on prior work (i.e. Dupas and Robinson 2013, "Why Don't the Poor Save More?"), we plan to test for heterogeneity based on (1) gender, (2) time preferences, and (3) family pressure (proxied by a measure of net transfers out of the household).

\section{$5 \quad$ Regression specifications}

\subsection{Level treatment effects from phone log data}

For the High-Frequency Phone Surveys, we will have several main specifications. First, we will compare the effects of having one (or more) mobile money account or lockbox, relative to the control.

$$
Y_{i s t}=\theta_{1} M M_{i}+\theta_{2} L B_{i}+\beta X_{i}+\mu_{s}+\epsilon_{i s t}
$$

where $Y_{i s t}$ is an outcome for individual $i$ at time $t$ in strata $s, \mu_{s}$ is a strata fixed effect, and $X_{i}$ are individual controls including a baseline measure of the outcome variable to improve precision (we plan to run regressions with and without these controls). We will test whether $\theta_{1}$ and $\theta_{2}$ are equal to 0 , as well as whether $\theta_{1}=\theta_{2}$. We are also interested in the effect of multiple accounts, which we can estimate as follows:

$$
Y_{i s t}=\alpha_{1} M M_{i}^{1}+\alpha_{2} M M_{i}^{\text {mult }}+\alpha_{3} L B_{i}^{1}+\alpha_{4} L B_{i}^{\text {mult }}+\beta X_{i}+\mu_{s}+\epsilon_{i s t}
$$

where $M M_{i}^{1}$ is a dummy for being offered 1 mobile money account, $M M_{i}^{\text {mult }}$ is a dummy for being offered multiple mobile money account, $L B_{i}^{1}$ is a dummy for being offered 1 lockbox, and $L B_{i}^{\text {mult }}$ is a dummy for being offered multiple lockboxes. We will test whether $\alpha_{1}=\alpha_{2}$ and whether $\alpha_{3}=\alpha_{4}$, as well as whether each of the $\alpha$ terms is equal to 0 . We also plan to run IV regressions (where usage of the account is instrumented with treatment) to show TOT effects, though we do not expect these to be primary specifications (we expect to instead focus on the ITT estimates).

Standard errors in all regressions will be clustered at the individual level.

\subsection{Responsiveness of shocks from phone log data}

We are interested in whether the accounts change people's ability to cope with shocks. We hypothesize that this effect could go either way. For instance, having access to savings instruments, and therefore, greater savings, may increase resilience. On the other hand, 
mental accounting may make people less likely to withdraw money from accounts in response to shocks. To examine this we will run regressions as follows:

$$
\begin{aligned}
Y_{i s t} & =\gamma_{1} M M_{i}+\gamma_{2} L B_{i}+\gamma_{3} S_{i t}+\gamma_{4} M M_{i} * S_{i t}+\gamma_{5} L B_{i} * S_{i t} \\
& +\beta X_{i}+\mu_{s}+\epsilon_{i s t}
\end{aligned}
$$

where $S_{i t}$ is one of several shocks we will measure (including own or household illness). We are interested in whether $\gamma_{4}$ and $\gamma_{5}$ are equal to 0 . The mental accounting effect may be more pronounced for those with multiple accounts. This will be examined through the following regression:

$$
\begin{aligned}
Y_{i s t} & =\theta_{1} M M_{i}^{1}+\theta_{2} M M_{i}^{\text {mult }}+\theta_{3} L B_{i}^{1}+\theta_{4} L B_{i}^{\text {mult }}+\theta_{5} S_{i t}+\theta_{6} S_{i t} * M M_{i}^{1} \\
& +\theta_{7} S_{i t} * M M_{i}^{\text {mult }}+\theta_{8} S_{i t} * L B_{i}^{1}+\theta_{9} S_{i t} * L B_{i}^{\text {mult }}+\beta X_{i}+\mu_{s}+\epsilon_{i s t}
\end{aligned}
$$

We will test whether $\theta_{6}=\theta_{7}$ and whether $\theta_{8}=\theta_{9}$, as well as whether each of the $\theta_{6-9}$ terms is equal to 0 .

In addition to the shocks experienced by our respondents over the normal course of events, we also provided some experimental variation in shocks to income by entering everyone in the phone-logs group into a lottery worth 2100 MWK (delivered in the form of airtime) on each day they were supposed to be called. We will examine how respondents in each group used this windfall, through the following regression:

$$
\begin{aligned}
Y_{i s t} & =\eta_{1} M M_{i}+\eta_{2} L B_{i}+\eta_{3} L_{i t}+\eta_{4} M M_{i} * L_{i t}+\eta_{5} L B_{i} * L_{i t} \\
& +\beta X_{i}+\mu_{s}+\epsilon_{i s t}
\end{aligned}
$$

where $L_{i t}$ is an indicator for whether someone won the lottery in the preceding week. We are interested in whether $\eta_{4}$ and $\eta_{5}$ are equal to 0 .

\subsection{Data from monitoring surveys}

For the follow-up surveys, our regression will be identical to those given by Equations (1) and (2). except that the outcome variables will be drawn from the monitoring surveys. In addition, we will use the following specification to measure the effects of the high-frequency phone surveys on behavior: 


$$
Y_{i s t}=\delta_{1} M M_{i}+\delta_{2} L B_{i}+\delta_{3} M M_{i} * P S_{i}+\delta_{4} L B_{i} * P S_{i}+\delta_{5} P S_{i}+\beta X_{i}+\mu_{s}+\epsilon_{i s t}
$$

where $P S_{i}$ is a dummy for being in the high-frequency phone survey treatment group. In addition to the tests listed in 5.1, we will test whether $\delta_{1}=\delta_{3}$, whether $\delta_{2}=\delta_{4}$, and whether $\delta_{5}=0$.

\section{References}

[1] Dupas, Pascaline and Jonathan Robinson (2013a). "Why Don't the Poor Save More? Evidence from Health Savings Experiments." American Economic Review 103 (4): 1138-1171. 